\title{
Ultrashort pulse induced nanogratings
}

\author{
Stefan Nolte ${ }^{1,2}$, Felix Zimmermann ${ }^{1}$, Sören Richter ${ }^{1}$, Anton Plech ${ }^{3}$, Ulf Peschel ${ }^{4}$, and Andreas Tünnermann ${ }^{1,2}$ \\ ${ }^{1}$ Institute of Applied Physics, Abbe Center of Photonics, Friedrich-Schiller-Universität Jena, Max-Wien-Platz 1,07734 Jena, Germany \\ ${ }^{2}$ Fraunhofer Institute of Applied Optics and Precision Engineering, Albert-Einstein-Straße 7, 07745 Jena, Germany \\ ${ }^{3}$ Institute for Synchrotron Radiation, Karlsruhe Institute of Technology, PO box 3640, 76021 Karlsruhe, Germany \\ ${ }^{4}$ Institute of Optics, Information and Photonics, Friedrich-Alexander-University Erlangen-Nürnberg, Günther-Scharowsky-Straße 1, building 24, \\ 91058 Erlangen, Germany \\ Author e-mail address: stefan.nolte@uni-jena.de
}

\begin{abstract}
When intense femtosecond laser pulses are focused into a glass substrate, selforganized periodic nanostructures, so-called nanogratings, are generated in a certain parameter regime. To clarify the ultimate structure of the nanogratings we employed focused ion beam (FIB) milling and small angle X-ray scattering (SAXS). The results considerably show that voids are the primary constituents and their number increases with ongoing exposure to laser pulses. Potential applications will be highlighted.
\end{abstract}

\section{Introduction}

About ten years ago grating like structures with periods smaller than the laser wavelength were observed inside fused silica after irradiation with tightly focused ultrashort laser pulses [1]. These self-organized, so-called "nanogratings" show a strong form birefringence due to their anisotropic structure. Emerging after several laser pulses [2] they are oriented perpendicular to the laser polarization (see Fig. 1(a)). By tuning the laser parameters the structural properties and thus the birefringence can be controlled $[3,4]$. Consequently, there are numerous potential applications from phase elements controlling the laser polarization $[3,4]$ to devices enabling the generation of optical vortices [5]. Nevertheless the self-organized formation process is not yet fully understood. One reason is that the actual structure of the nanogratings is difficult to explore. Due to the small feature size optical microscopy cannot be used and electron microscopic investigations require sample preparations leading to blurring and distortion of essential structure details. Recent work on cleaved samples revealed nanoporous fine structures and planar nanocracks within nanogratings [6].

We employed small angle X-ray scattering (SAXS) as well as focused ion beam (FIB) milling and scanning electron microscopy (SEM) to determine the principal constituents of nanogratings [7].

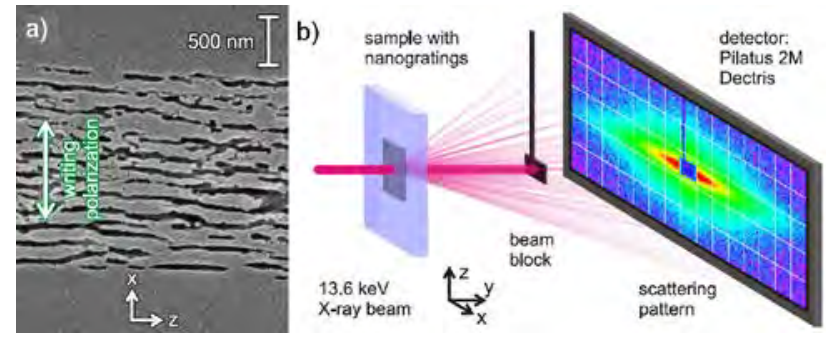

Fig. 1. SEM micrograph of gratings after etching with hydrofluoric acid (a) and schematic of the SAXS measurement (b).

\section{Methodology}

Nanogratings are inscribed using the frequency doubled $(515 \mathrm{~nm}$ ) light of a mode locked laser (Amplitude Systemes, t-pulse 500). This laser provides pulses with a duration of about $450 \mathrm{fs}$, pulse energies up to $200 \mathrm{~nJ}$ at repetition rates up to $10 \mathrm{MHz}$. The repetition rate was reduced by an external acusto-optical modulator in order to avoid heat accumulation effects. For focusing we used an aspheric lens (New Focus 5722) with a numerical aperture of 0.55 .

In order to explore the nanograting structure in a nondestructive way, we studied x-ray diffraction. Thereby no preparation steps are required. Samples with nanogratings were illuminated with a probe beam (photon energy 13.6 keV, cSAXS beamline at the Swiss Light Source (Villigen, $\mathrm{CH}$ )) that is scattered and the pattern is recorded with a large direct converting pixel converter (see Fig. 1(b)). In addition, FIB milling has been used in order to dissect a volume containing nanogratings into $12 \mathrm{~nm}$ slices. After each milling step high-resolution images have been taken by SEM. 


\section{Results}

The SAXS scattering patterns reveal different structural dimensions along the $\mathrm{x}$ and $\mathrm{z}$ direction. The evaluation along $\mathrm{x}$ (direction of laser polarization) showed a distribution whereby two distinct populations with different diameters exist. As it can be seen in Fig. 2(a) these are roughly independent of pulse energy and number of laser pulses. This indicates that once the structure is formed no further growth along the laser polarization takes place. Fig. 2(b) shows the aspect ratio between the structure size along the $\mathrm{z}$ - and $\mathrm{x}$-axis. The aspect ratio and thus the structure size along the $\mathrm{z}$-direction increases only during the first $\approx 100$ pulses, afterwards it stays constant.
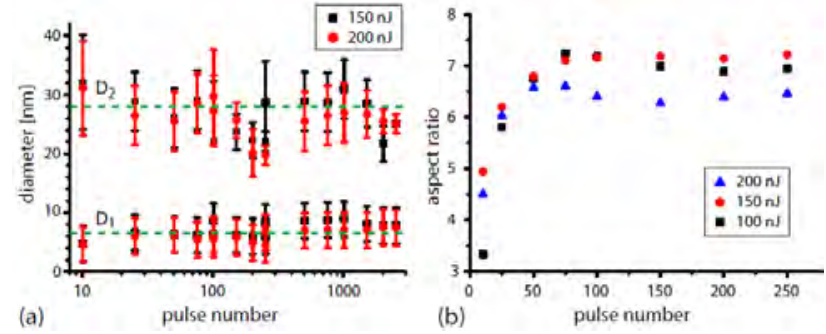

Fig. 2. Results of the SAXS measurements.

In addition, the SAXS data show an increase of the characteristic scattering parameter with rising pulse number. This can be attributed to a growing number of pores that corresponds well to the observed decrease in the grating period during prolonged laser exposure $[3,4]$.
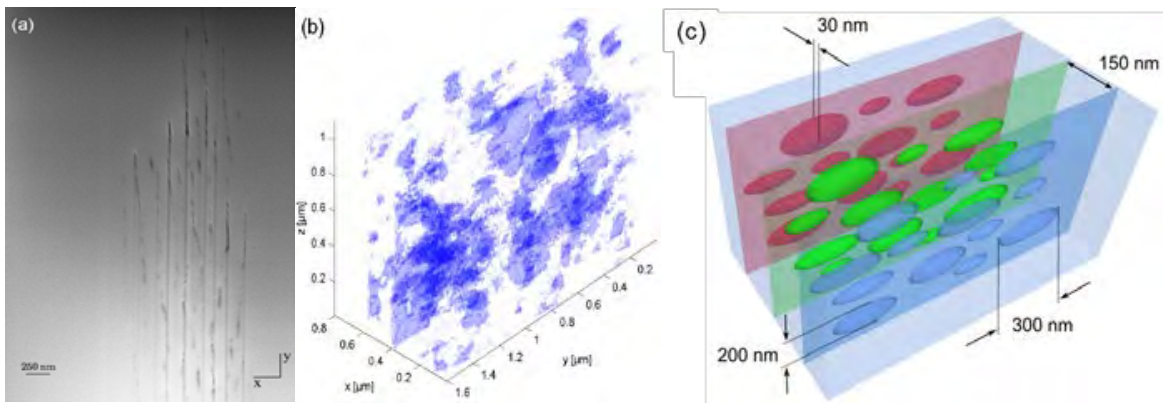

Fig. 3. SEM micrograph of a FIB-slice in the x-y-plane (a) and 3D plot of nanogratings constructed out of many FIB slices (b).

(c) Sketch illustrating typical dimensions.

To obtain further insight into the real structure of nanogratings we performed FIB measurements. Figure 3(a) shows an SEM micrograph of a FIB slice that depicts the micro porous character of the grating. With an appropriate Fourier filtering algorithm we isolated the pores from hundred FIB slices in order to obtain a 3D view of their size (see Fig. 3(b)). In accordance to the SAXS measurements, the transverse dimensions of the pores (200-300 nm) are significantly larger than their thickness $(30 \mathrm{~nm})$, see Fig. 3(c).

\section{Acknowledgements}

We gratefully acknowledge financial support from Deutsche Forschungsgemeinschaft DFG (priority program 1327). Furthermore, we thank the Swiss Light Source (cSAXS beamline at Paul Scherrer Institute, Villigen, CH) for the beamtime. Sören Richter acknowledges supported by the Hans L. Merkle Stiftung.

\section{References}

(1)

[1] Y. Shimotsuma, P.G. Kazansky, J. Qiu and K. Hirao, "Nanostructuring of transparent materials by ultrashort light pulses," Phys. Rev. Lett. 91, 247405 (2003).

[2] S. Richter, M. Heinrich, S. Döring, A. Tünnermann, and S. Nolte, "Formation of Femtosecond Laser-Induced Nanogratings at High Repetition Rates", Appl. Phys. A 104, 503-507 (2011).

[3] L.P.R. Ramirez, M. Heinrich, S. Richter, F. Dreisow, R. Keil, A.V. Korovin, U. Peschel, S. Nolte, and A. Tünnermann, "Tuning the Structural Properties of Femtosecond-Laser-Induced Nanogratings", Appl. Phys. A 100, 1-6 (2010).

[4] S. Richter, M. Heinrich, S. Döring, A. Tünnermann, S. Nolte, U. Peschel, "Nanogratings in fused silica: Formation, control, and applications," J. Laser Appl., 24(4), 4020081-4020088 (2012).

[5] M. Beresna, M. Gecevičius, P.G. Kazansky, and T. Gertus, "Radially polarized optical vortex converter created by femtosecond laser nanostructuring of glass", Appl. Phys. Lett. 98, 201101 (2011).

[6] R. Taylor, C. Hnatovsky, and E. Simova, "Applications of femtosecond laser induced self-organized planar nanocracks inside fused silica glass," Laser \& Photon. Rev. 2, 26-46 (2008).

[7] S. Richter, A. Plech, M. Steinert, M. Heinrich, S. Döring, F. Zimmermann, U. Peschel, E.-B. Kley, A. Tünnermann, S. Nolte, "On the fundamental structure of femtosecond laser-induced nanogratings," Laser Photon. Rev., 6(6), 787-792 (2012). 